Published in final edited form as:

Clin Cancer Res. 2009 December 1; 15(23): 7421-7428. doi:10.1158/1078-0432.CCR-09-1691.

\title{
Phase II Study of Dasatinib in Patients with Metastatic Castration-Resistant Prostate Cancer
}

\author{
Evan Y. Yu ${ }^{1}$, George Wilding ${ }^{2}$, Edwin Posadas ${ }^{3}$, Mitchell Gross ${ }^{4}$, Stephane Culine ${ }^{5}$, \\ Christophe Massard $^{6}$, Michael J. Morris ${ }^{7}$, Gary Hudes ${ }^{8}$, Fabio Calabrò ${ }^{9}$, Shinta Cheng ${ }^{10}$, \\ Géralyn C. Trudel ${ }^{10}$, Prashni Paliwal ${ }^{10}$, and Cora N. Sternberg ${ }^{9}$ \\ 1 University of Washington, Seattle, Washington \\ 2University of Wisconsin Carbone Cancer Center, Madison, Wisconsin \\ ${ }^{3}$ University of Chicago, Chicago, Illinois \\ ${ }^{4}$ Cedars-Sinai Medical Center, Los Angeles, California \\ ${ }^{5}$ CRLC Val d'Aurelle, Montpellier, France \\ ${ }^{6}$ Institut Gustave Roussy, Villejuif, France \\ ${ }^{7}$ Memorial Sloan-Kettering Cancer Center, New York, New York \\ ${ }^{8}$ Fox Chase Cancer Center, Philadelphia, Pennsylvania \\ ${ }^{9}$ San Camillo Forlanini Hospital, Rome, Italy \\ ${ }^{10}$ Bristol-Myers Squibb, Wallingford, Connecticut
}

\section{Abstract}

Purpose-Antiproliferative and antiosteoclastic activity from preclinical models show potential for dasatinib, an oral SRC and SRC family kinase inhibitor, as a targeted therapy for patients with prostate cancer. This phase II study investigated the activity of dasatinib in patients with metastatic castration-resistant prostate cancer (CRPC).

\begin{abstract}
Experimental Design-Chemotherapy-naive men with CRPC and increasing prostate-specific antigen were treated with dasatinib 100 or $70 \mathrm{mg}$ twice daily. Endpoints included changes in prostate-specific antigen, bone scans, measurable disease (Response Evaluation Criteria in Solid Tumor), and markers of bone metabolism. Following Prostate Cancer Working Group 2 guidelines, lack of progression according to Response Evaluation Criteria in Solid Tumor and bone scan was determined and reported at 12 and 24 weeks.
\end{abstract}

Results-Forty-seven patients were enrolled and received dasatinib (initial dose $100 \mathrm{mg}$ twice daily, $n=25 ; 70 \mathrm{mg}$ twice daily, $n=22)$, of whom $41(87 \%)$ had bone disease. Lack of

Copyright $\odot 2009$ American Association for Cancer Research.

Requests for reprints: Cora N. Sternberg, Department of Medical Oncology, San Camillo Forlanini Hospital, Nuovi Padiglioni, 4th floor, Circonvallazione Gianicolense 87, Rome 00152, Italy. Phone: 39-06-5870-4356; Fax: 39-06-663-0771; cstern@ mclink.it.

Note: E.Y. Yu, G. Wilding, and M.J. Morris are members of the Prostate Cancer Clinical Trials Consortium sponsored by the Department of Defense. Prior presentations: 2008 ASCO Annual Meeting, Chicago, IL, May 2008. J Clin Oncol 26: 2008 (Suppl; abstract 5156).

Clinical trials registry number: NCT00385580.

Disclosure of Potential Conflicts of Interest

S. Cheng, G.C. Trudel, and P. Paliwal are employed by Bristol-Myers Squibb; S. Cheng and G.C. Trudel have ownership interests in Bristol-Myers Squibb; G. Wilding and M. Gross have received commercial research support from Bristol-Myers Squibb; G. Wilding is a consultant for Bristol-Myers Squibb. 
progression was achieved in $20(43 \%)$ patients at week 12 and in $9(19 \%)$ patients at week 24 . Of 41 evaluable patients, $21(51 \%)$ patients achieved $\geq 40 \%$ reduction in urinary $\mathrm{N}$-telopeptide by week 12, with $33(80 \%)$ achieving some level of reduction anytime on study. Of 15 patients with elevated urinary N-telopeptide at baseline, 8 (53\%) normalized on study. Of 40 evaluable patients, $24(60 \%)$ had reduction in bone alkaline phosphatase at week 12 and $25(63 \%)$ achieved some reduction on study. Dasatinib was generally well tolerated and treatment-related adverse events were moderate.

Conclusions-This study provides encouraging evidence of dasatinib activity in bone and reasonable tolerability in chemotherapy-naive patients with metastatic CRPC.

Prostate cancer is the most common cancer, with high mortality rates, affecting men in the United States and Europe $(1,2)$. The natural progression of prostate cancer that is not cured with local therapy results in metastases, most commonly to bone, and resistance to androgen deprivation therapy. Second-line hormonal therapies (steroidal/nonsteroidal antiandrogens, ketoconazole, and diethylstilbestrol) may be added to luteinizing hormone-releasing hormone agonists or orchiectomy; however, this produces a relatively short response in less than half of castrate patients (3). No second-line hormonal therapies have been associated with an improvement in survival. Additionally, patients with castration-resistant prostate cancer (CRPC) with bone metastases are often treated with bisphosphonates to prevent skeletal-related events $(4,5)$.

Docetaxel chemotherapy is used in men with metastatic CRPC and has been shown to extend survival (6-8). However, it is associated with adverse events, including fatigue, myelo-suppression, and neuropathy, toxicities that may not be acceptable to asymptomatic patients, and as such, the optimal timing for starting chemotherapy remains uncertain. For both the patient and the physician, delaying the need for chemotherapy is an important goal. Furthermore, bone metastases are responsible for substantial morbidity in patients with advanced prostate cancer, and new agents that target bone metastases warrant evaluation.

Several lines of evidence indicate a role for SRC family kinases (SFK) in prostate cancer (9, 10). These include SFK over-expression in prostate cancer cell lines and tissues and reduced cancer cell proliferation, invasion, and migration following SRC inhibition (9, 11-14). Furthermore, SFKs play an important role in osteoclast and osteoblast function, with SRC inhibition delaying the appearance and decreasing the size of bone metastases in murine models of breast cancer (15-17). SRC may be involved in androgen-independent growth during advanced stages of disease. Mendiratta et al. have shown that prostate cancer cell lines exhibiting low androgen receptor activity by transcriptional profiling exhibit high SRC activity and sensitivity to dasatinib (18).

Dasatinib (SPRYCEL; Bristol-Myers Squibb) is an oral tyrosine kinase inhibitor with potent activity against the SFKs, BCR-ABL, platelet-derived growth factor receptor, and c-KIT (19-22). Dasatinib is currently used as an ABL kinase inhibitor for the treatment of chronic myelogenous leukemia (23-25) and acute lymphoblastic leukemia (26). Several non-SFK targets of dasatinib are also hypothesized to play a role in prostate cancer. EphA2 is upregulated in prostatic intraepithelial neoplasia and prostate cancer and is implicated in the malignant transformation of breast and prostate cancer cells $(27,28)$. Platelet-derived growth factor receptor- $\beta$ may also play a role in a subset of prostate cancer metastatic to bone (29).

Several preclinical studies have highlighted the ability of dasatinib to inhibit proliferation of prostate cancer cells $(20,21,30)$, reduce prostate cancer xenograft growth $(19,30)$, and inhibit development of lymph node metastases in both androgen-sensitive and androgenresistant tumors (30). Dasatinib also reduces osteoclast proliferation and calcium release in 
bone resorption assays (31). Dasatinib treatment of orthotopic murine bone tumor models have shown decreased prostate-specific antigen (PSA), increased bone mineral density, decreased serum calcium, and potentiated docetaxel chemotherapy effects (32). Overall, these preclinical data provided the rationale for testing dasatinib in the clinical setting for the treatment of patients with metastatic prostate cancer.

Therefore, a phase II study (CA180085) was conducted to evaluate the efficacy and safety of dasatinib in patients with metastatic CRPC and increasing PSA levels. We report results from patients initially treated with dasatinib at a dose of either 100 or $70 \mathrm{mg}$ orally twice daily. Although the trial protocol was designed before the availability of recent criteria for prostate cancer trials in CRPC [Prostate Cancer Working Group 2 (PCWG2); ref. 33], the data have been analyzed and presented where possible according to these updated guidelines.

\section{Materials and Methods}

\section{Study design and treatments}

CA180085 was a phase II, open-label study conducted at 10 centers in the United States and Europe (Italy and France) in men with metastatic CRPC. Patients were initially administered dasatinib $100 \mathrm{mg}$ orally twice daily. After the first 25 patients were enrolled, the dosage was reduced to $70 \mathrm{mg}$ twice daily for subsequent patients $(n=22)$. This change occurred, in part, due to observations in other clinical trials with dasatinib where lower doses of dasatinib resulted in equal efficacy but improved tolerability (34). Additionally, grade 2 pleural effusions were reported in $3(15 \%)$ of the first 20 patients treated at $100 \mathrm{mg}$ twice daily. Of this initial patient group, 3 of $20(15 \%)$ patients also had at least one dose reduction necessary and 6 of $20(30 \%)$ had at least one dose interruption for toxicity. For subjects in both dosage groups, doses could be reduced, interrupted, or stopped as necessary for toxicity until only grade $\leq$ toxicities were present and any grade 2 toxicities were managed with outpatient therapy or deemed acceptable by the physician and patient.

Patients who initially started on dasatinib $100 \mathrm{mg}$ twice daily could have dose modification to $70 \mathrm{mg}$ twice daily, followed by $50 \mathrm{mg}$ twice daily, and then $100 \mathrm{mg}$ once daily as necessary. Patients who initially started on $70 \mathrm{mg}$ twice daily were allowed dose modifications to $50 \mathrm{mg}$ twice daily and $100 \mathrm{mg}$ once daily as necessary. Dose reescalation after prior reduction was permitted at investigator discretion. Dose interruptions were required for any drug-related toxicities of grade $\geq 3$ and were allowed for clinically unacceptable drug-related grade 2 toxicities.

\section{Patient eligibility}

The key inclusion criteria for this trial included males age $\geq 18$ years with histologically or cytologically proven prostate carcinoma and radiologic evidence of metastatic disease. Progressive disease was mandatory before entry and was defined as two serially increasing PSA levels of any quantity, obtained $\geq 1$ week apart with castrate serum testosterone levels $(<50 \mathrm{ng} / \mathrm{dL})$. Required treatment withdrawal time frames for study enrollment were 4 weeks from anti-androgens ( 6 weeks in the case of bicalutamide) or adrenal androgen production inhibitors, 6 weeks from isotopes (12 weeks in the case of strontium- 89 or immunotherapy), and/or 4 weeks from major surgery. Other requirements included adequate organ function and an Eastern Cooperative Oncology Group performance status 0 to 2 with life expectancy 23 months.

Key exclusion criteria included symptomatic central nervous system metastases, a medical condition that could significantly increase the risk of toxicity (coagulation disorders, infection requiring intravenous antibiotics, gastrointestinal bleeding, and/or clinically 
significant cardiovascular disease), pleural or pericardial effusion, prior or ongoing treatment with cytotoxic chemotherapy, glucocorticoids with antitumor intent, history of tumor vaccines or immunostimulating therapy, or history of another malignancy within the preceding 5 years (except in situ nonmelanomatous carcinoma of the skin). Potent CYP3A4 inhibitors and QTc prolonging agents were also prohibited while on study. Concomitant luteinizing hormone-releasing hormone agonists were continued (if applicable) to maintain castrate levels of testosterone ( $<50 \mathrm{ng} / \mathrm{dL})$, and patients who were already on bisphosphonates were allowed to continue use throughout the study. Treatment with bisphosphonates could not commence within 3 weeks of study entry or while on study.

Patients were treated with dasatinib until progression of disease (defined below), unacceptable toxicity at the lowest allowable dose reduction (100 $\mathrm{mg}$ once daily) despite optimal supportive care, withdrawal of informed consent, or investigator recommendation.

The study was approved by the ethics committees of all participating sites, and all patients signed written informed consent before commencement of study procedures.

\section{Evaluation of clinical activity}

Serum PSA levels were measured at the screening visit and every 4 weeks on study. PSA response was defined as $\geq 50 \%$ decrease in PSA from baseline for two consecutive evaluations $\geq 2$ weeks apart. PSA doubling time was calculated in patients with $\geq 2$ pretreatment PSA values and $\geq 2$ on-study PSA values and was defined as $\log _{2}$ divided by the slope of the log PSA line. Serial bone scans were obtained approximately every 12 weeks and received designation as disappearance of lesion(s), stable, or appearance of new lesion(s). Investigators at each site performed tumor assessments for patients with measurable lesions at baseline and every 12 weeks thereafter using modified Response Evaluation Criteria in Solid Tumor (RECIST) criteria (35). Analysis of the tumor measurements and determination of response excluded pelvic lymph nodes $<2 \mathrm{~cm}$ in size as per PCWG2 recommendations (33).

Serum bone alkaline phosphatase (BAP) and urinary N-telopeptide (uNTX) levels were measured at the screening visit and every 4 weeks on study to assess for treatment-related changes in bone metabolism. To capture potentially meaningful changes, a $\geq 40 \%$ decrease in UNTX at week 12 from baseline is reported. This value was based on the findings from a previous report in men with CRPC that described a 40\% decrease in UNTX at 3 months as resulting in a significant $(17 \%)$ reduction in the risk of death regardless of baseline level (36). Additionally, because treatment-associated normalization of elevated uNTX has been shown to correspond with a 59\% reduction in death and $49 \%$ increase in skeletal-related event-free survival in men with CRPC (36), the proportion of patients with baseline uNTX above the upper limit of normal ( $63 \mathrm{nmol} / \mathrm{L}$ creatinine) who had a uNTX reduction to within normal limits (3-63 nmol/L creatinine) is also presented.

The primary study endpoint was a composite response rate [subsequently called in this article as composite response/stable disease (SD) rate] developed before PCWG2 and defined as either confirmed $\geq 50 \%$ PSA decline; SD, confirmed complete response, or confirmed partial response using RECIST; or confirmed disappearance of lesion(s) by radionuclide bone scan assessment. However, in light of PGCW2 recommendations that phase II trials should alter the focus from measurement of response to determination of progression, the data have also been analyzed and reported in this manner. RECIST response and SD were both considered in the definition of lack of progression. PSA changes were not included as a marker of response or progression and are reported separately. Additionally, lack of progression by bone scan was assessed in combination with RECIST, when assessable, at multiple designated time points. 
Progression was defined as tumor progression by RECIST or by at least one definite new lesion on bone scan, even without additional confirmation. Separate from this analysis, the study protocol allowed patients with asymptomatic increases in PSA or appearance of new lesions on bone scan before 24 weeks, without other evidence of progression (RECIST or clinical symptoms), to continue therapy.

Secondary efficacy endpoints include site investigator assessment of changes in bone scans, RECIST, PSA, and bone turnover markers (BAP and uNTX).

\section{Safety assessments}

Patients underwent a physical examination, Eastern Cooperative Oncology Group performance status, chest X-ray, electrocardiogram, echocardiogram, vital signs, signs and symptoms, clinical chemistry, and coagulation assessment at screening. Vital signs and clinical chemistry were reevaluated at week 2 then, along with performance status, every 4 weeks until the end of treatment. A chest X-ray was re-performed at week 4 , and electrocardiogram and echocardiograms were re-performed at weeks 2, 8, and 12. Coagulation parameters were measured again at week 12 . Adverse events were evaluated on an ongoing basis throughout the study and classified according to the National Cancer Institute Common Toxicity Criteria (version 3.0).

\section{Statistical analyses}

The original study hypothesis was that dasatinib would have a composite response/SD rate of at least $10 \%$. Thus, a modified Gehan two-stage design was used. If none of the first 29 subjects responded or had at least SD at week 12, the true composite response/SD rate was unlikely to be $>10 \%$ and the study would close to accrual. The analysis was positive; therefore, additional subjects were enrolled, for a total accrual of 47 subjects.

Analysis of efficacy and safety was performed following a database lock in November 2008 for patients treated with twice daily dasatinib, combining data from both 100 and $70 \mathrm{mg}$ twice daily initial dosing groups. Both analyses included all patients who received at least one dose of dasatinib. A two-sided 95\% exact confidence interval for composite response/ SD rate was calculated. Lack of progression was evaluated at 12 and 24 weeks depending on availability of assessments.

\section{Results}

\section{Patients and treatment}

Forty-seven patients were enrolled in the study ( 25 initially received dasatinib $100 \mathrm{mg}$ twice daily and 22 initially received dasatinib $70 \mathrm{mg}$ twice daily). All 47 patients were assessed for toxicity and lack of progression. Forty-three patients were evaluable for PSA decline. All patients underwent bone scans at baseline, and 41 patients had bone metastases identified. Thirty-nine patients underwent an additional bone scan at week 12 . Twenty-three patients had RECIST-evaluable lesions at baseline; 19 had at least one on-study RECIST assessment. Bone markers were evaluable for uNTX $(n=41)$ and BAP $(n=40)$. Patient characteristics and demographic data are presented in Table 1. Treatment dosing and discontinuation information is summarized in Table 2.

\section{Efficacy}

As reported previously (37), an original predefined primary endpoint of composite response/ SD rate was achieved in 13 of 47 (28\%) patients (95\% confidence interval, 16-43\%), with $95 \%$ confidence interval above the minimum anticipated response rate of $10 \%$. The PCWG2-defined endpoint of lack of progression by both RECIST and bone scan analysis 
was met by 20 of 47 (43\%) patients at week 12 and by 9 of 47 (19\%) patients at week 24 . Of the 20 patients who achieved the 12-week lack of progression endpoint, 11 patients were treated initially with dasatinib $100 \mathrm{mg}$ twice daily and 9 were treated initially with dasatinib $70 \mathrm{mg}$ twice daily. Of the 9 patients who achieved the 24-week lack of progression endpoint, 3 patients were initially treated with dasatinib $100 \mathrm{mg}$ twice daily and 6 were initially treated with dasatinib $70 \mathrm{mg}$ twice daily.

Forty-one patients had bone scan detected bone metastases at baseline. Thirty-nine underwent on-study bone scan assessments at weeks 12 and 14 patients remained on-study for additional assessment at week 24 . Twenty-two patients at week 12 were reported as having no new lesions. Further, one patient had a cranial lesion that was undetectable at subsequent assessments. Overall, 23 (49\%) patients at week 12 had no new bone lesions. At week 24,9 of the remaining 14 patients assessed still had no new bone lesions.

Twenty-three patients had RECIST-evaluable lesions at baseline (excluding those with lymph nodes in the pelvis $<2 \mathrm{~cm}$, as per PCWG2). The disease control rate for patients with measurable lesions by RECIST (complete response + partial response + SD) was 12 of 23 (52\%) after 12 weeks. All 12 patients had SD using RECIST. Seven (30\%) RECISTevaluable patients had progressive disease. The remaining 4 patients had incomplete data or no on-study tumor assessment and were not evaluable. After 24 weeks, the disease control rate by RECIST was 6 of 23 (26\%); of the 12 RECIST-evaluable patients that remained on study beyond 12 weeks, 2 patients had progressive disease ( 1 by RECIST and 1 on bone scan), 1 patient discontinued for increasing PSA, 1 had investigator defined clinical progression, and 2 discontinued for toxicity. Maximum changes in tumor measurements are presented in Fig. 1A.

Three patients had a PSA decline of $\geq 50 \%$. One patient had a confirmed sustained PSA response. He is currently at a dose of $70 \mathrm{mg}$ twice daily and remains on dasatinib, now $>80$ weeks (at time of article preparation), with a persistent PSA response. This patient has developed no new lesions by both CT and bone scan. The PSA doubling time was prolonged in 34 of $43(79 \%)$ assessable patients. Maximum PSA changes from baseline are presented in Fig. 1B.

Because prognostic significance with the 3-month uNTX level has been reported (36), we evaluated this as a potential treatment-response biomarker. The proportion of patients who achieved a $\geq 40 \%$ reduction in uNTX by week 12 was 21 of $41(51 \%)$ : 11 of $21(52 \%)$ for those receiving bisphosphonates and 10 of $20(50 \%)$ for those not receiving bisphosphonates. This analysis included patients who had abnormal or normal values at baseline.

Of note, the proportion of patients who achieved any decline of uNTX while on study was 33 of $41(80 \%)$. The maximal change in uNTX level for each patient is presented in Fig. 2A.

At baseline, 15 patients had above normal levels of uNTX. Of these patients, 3 of 4 (75\%) on bisphosphonates achieved a reduction in uNTX levels to within normal range, and of those patients who were not on bisphosphonates, 5 of $11(45 \%)$ achieved a reduction in uNTX levels to within normal range (Fig. 2B).

Forty patients had baseline BAP levels. Any reduction in BAP levels by week 12 was reported in 24 of $40(60 \%)$ patients. Of these, reductions in BAP were achieved in 11 of 21 (52\%) patients who were receiving bisphosphonates and 13 of $19(68 \%)$ patients who were not receiving bisphosphonates. 
Twenty-five of 40 (63\%) patients achieved some decrease in BAP from baseline while on study. The maximal change in BAP from baseline for individual patients, according to whether or not they were receiving bisphosphonates, is presented in Fig. 3.

\section{Safety and tolerability}

An amendment in dasatinib dosing from 100 to $70 \mathrm{mg}$ twice daily was made in an attempt to decrease the incidence of pleural effusions. However, on analysis, there was no significant difference between the two cohorts, and pleural effusions (any grade) were reported in 51\% of the overall population. Pericardial effusions were also similar between dosing cohorts, occurring in $23 \%$ of all patients (grade 1 only). No unique rules for dose reductions or interruptions were for made for pleural or pericardial effusions and general study rules for adverse events were applied. Aside from pleural effusion, the most common adverse events were diarrhea (62\%), nausea (47\%), and fatigue (45\%). Overall, adverse events were shown to be moderate and similar in both dosing cohorts and are reported together in Table 3. Most adverse events experienced were grade $1 / 2$ in severity. This included 15 of 47 (32\%) patients who developed grade $1 / 2$ hypocalcemia, none of which worsened with ongoing dasatinib treatment. Adverse events were generally reversible and manageable with dose interruptions or reductions. Reasons for discontinuation, dose reduction (57\%) and dose interruption (60\%), are presented in Table 3. At the last follow-up (November 2008), 2 patients remained on dasatinib (both $>1$ year). The median duration of therapy was 2.8 months (range 0.03-13.8 months). Two disease-related deaths have been reported. Neither death was treatment-related and both occurred $\geq 30$ days after discontinuation of dasatinib.

\section{Discussion}

Bone morbidity is a serious, debilitating complication for patients with CRPC. In this study, single-agent, twice daily dasatinib showed biological activity in chemotherapy-naive patients with CRPC as evidenced principally by bone biomarkers. Dasatinib produced reduction in BAP and uNTX in the majority of patients. Impressively, a significant proportion of men previously and concurrently receiving bisphosphonates had additional reduction in bone biomarkers in response to treatment with dasatinib. This is consistent with the proposed mechanism of action for dasatinib. Inhibition of SRC reduces osteoclast activity and results in inhibition of osteolysis. These data offer early hints that dasatinib may have bone effects in addition to that of bisphosphonates.

Beyond survival, there are other outcomes that are clinically important for patients with metastatic CRPC. Reduction of skeletal-related events and bone pain associated with bone metastases is an important goal, leading to significant improvement in quality of life. uNTX correlates with presence and extent of bone metastases (38), and an elevated uNTX level is an independent prognostic factor of reduced overall survival in patients with bone metastases from prostate cancer $(9,39-43)$. Normalization of uNTX is associated with a lower incidence of fractures, lower rate of bony disease progression, palliative response, and improved overall survival (36). In a subset analysis of CRPC patients, a $40 \%$ decrease in uNTX at 3 months resulted in $\sim 17 \%$ reduction in the risk of death regardless of baseline uNTX levels (36). Although not validated as a surrogate marker for prostate cancer endpoints, changes in uNTX levels in response to therapy with dasatinib show signs of biological activity.

BAP also has important prognostic potential and offers information on osteoblastic activity separate from that provided by uNTX on osteoclastic activity. In an analysis of three large randomized trials of patients with bone metastases, high levels of BAP were associated with a significant increase in the risk of skeletal-related events compared with low BAP, and correlation was strongest for patients with prostate cancer (40). In a study of 31 patients with 
CPRC treated with docetaxel and atrasentan, an endothelin-A receptor antagonist, BAP, significantly declined with therapy and increased at the time of progression (44). Thus, in this study, dasatinib has shown effects on both osteoclastic and osteoblastic components of bone disease in prostate cancer, offering important hints of clinical relevance.

The bone effects and potential antitumor activity of dasatinib in prostate cancer are due, at least in part, to inhibition of SRC and other SFKs. Phase I studies have shown that exposures of dasatinib required to substantially inhibit SRC activity are achieved in patients with solid tumors (45). This study is the first clinical trial, to our knowledge, that shows inhibition of SRC to have biological activity through decrease in bone turnover biomarkers. Thus, dasatinib may have a future role for patients with prostate cancer as both an antitumor and a bone-targeted agent. We plan to further evaluate bone biomarkers and skeletal-related events as major endpoints in upcoming studies. Additionally, ongoing efforts are evaluating the efficacy of dasatinib in combination with docetaxel in CRPC. This is supported by preclinical models where dasatinib has synergistic effects with taxanes $(19,32)$. Early data from an ongoing phase I/II study are promising (46), and a large phase III, randomized, placebo-controlled trial of docetaxel with dasatinib is ongoing.

The original primary study endpoint, which was a composite response rate, was developed before PCWG2. However, in light of PCWG2 guidelines that phase II trials should expand the focus from measurement of response to determination of progression, we have also presented the lack of progression endpoint.

The original progression criteria also did not acknowledge new bone scan lesions in the absence of other clinical evidence of progression. Although there is much controversy and lack of standardization in reporting bone scan progression, PCWG2 recommends that it should be defined as two or more new lesions, confirmed by two or more new lesions on subsequent bone scans performed $>6$ weeks later. Because data on the number of new bone lesions were not collected and confirmatory bone scans were not always performed (as per the study protocol), we were unable to adopt this into our lack of progression analysis. As a result, the requirement for lack of progression in the current report is more stringent than PCWG2, as any new bone scan lesion was considered progression without additional confirmation.

In this study, single-agent dasatinib was reasonably well tolerated, with the majority of adverse events being mild or moderate and reversible with dose reduction or interruption. Although the development of pleural effusions was concerning in this patient population, subsequent reports of dasatinib used in combination with docetaxel in patients with metastatic CRPC reported only $7 \%$ of the entire population as developing pleural effusions (46). Although the biological mechanism is yet uncertain, the utilization of corticosteroids with docetaxel administration may have prevented the development of pleural effusion. Additionally, dasatinib was administered at a dose of $100 \mathrm{mg}$ once daily rather than twice daily dosing. In hematologic malignancies, dasatinib $100 \mathrm{mg}$ once daily provides responses similar to those in patients taking the approved $70 \mathrm{mg}$ twice daily dose, with reduced incidence of side effects (34). It will be interesting to see if similar findings hold true in this population of prostate cancer patients, as subsequent patients $(n=47)$ in an ongoing study are being treated at a $100 \mathrm{mg}$ daily dose.

In conclusion, the analysis of this study provides encouraging evidence of the activity and tolerability of dasatinib in chemotherapy-naive patients with metastatic CRPC. Our data with bone turnover biomarkers is the first to emphasize this unique mechanism of action from dasatinib-induced inhibition of SRC. It also introduces a novel therapy that may offer beneficial effects targeted to bone with the potential for dual antitumor activity. Dasatinib 
warrants further evaluation with both antitumor and skeletal morbidity endpoints, and efforts are ongoing.

\section{Acknowledgments}

Grant support: Department of Defense Prostate Cancer Clinical Trials Consortium awards W81XWH-07-1-0097, W81XWH-06-1-0258, and W81XWH-06-1-0241; Prostate Cancer Foundation Therapy Consortium; and BristolMyers Squibb. Editorial assistance for this article was provided by StemScientific and funded by Bristol-Myers Squibb.

\section{References}

1. Ferlay J, Autier P, Boniol M, Heanue M, Colombet M, Boyle P. Estimates of the cancer incidence and mortality in Europe in 2006. Ann Oncol. 2007; 18:581-92. [PubMed: 17287242]

2. Jemal A, Siegel R, Ward E, et al. Cancer statistics, 2008. CA Cancer J Clin. 2008; 58:71-96. [PubMed: 18287387]

3. Scher HI, Sawyers CL. Biology of progressive, castration-resistant prostate cancer: directed therapies targeting the androgen-receptor signaling axis. J Clin Oncol. 2005; 23:8253-61. [PubMed: 16278481]

4. Saad F, Gleason DM, Murray R, et al. A randomized, placebo-controlled trial of zoledronic acid in patients with hormone-refractory metastatic prostate carcinoma. J Natl Cancer Inst. 2002; 94:145868. [PubMed: 12359855]

5. Saad F, Chi K, Fleshner N. The role of bisphosphonates in the management of bone metastases in prostate cancer. Can J Urol. 2004; 11:2376-82. [PubMed: 15576002]

6. Berthold DR, Pond GR, Soban F, de Wit R, Eisenberger M, Tannock IF. Docetaxel plus prednisone or mitoxantrone plus prednisone for advanced prostate cancer: updated survival in the TAX 327 study. J Clin Oncol. 2008; 26:242-45. [PubMed: 18182665]

7. Petrylak DP, Tangen CM, Hussain MH, et al. Docetaxel and estramustine compared with mitoxantrone and prednisone for advanced refractory prostate cancer. N Engl J Med. 2004; 351:1513-20. [PubMed: 15470214]

8. Tannock IF, de Wit R, Berry WR, et al. Docetaxel plus prednisone or mitoxantrone plus prednisone for advanced prostate cancer. N Engl J Med. 2004; 351:1502-12. [PubMed: 15470213]

9. Fizazi K. The role of Src in prostate cancer. Ann Oncol. 2007; 18:1765-73. [PubMed: 17426060]

10. Thomas X, Olteanu N, Charrin C, Lheritier V, Magaud JP, Fiere D. Acute lymphoblastic leukemia in the elderly: the Edouard Herriot Hospital experience. Am J Hematol. 2001; 67:73-83. [PubMed: 11343378]

11. Chang YM, Kung HJ, Evans CP. Nonreceptor tyrosine kinases in prostate cancer. Neoplasia. 2007; 9:90-100. [PubMed: 17357254]

12. Edwards J, Krishna NS, Witton CJ, Bartlett JM. Gene amplifications associated with the development of hormone-resistant prostate cancer. Clin Cancer Res. 2003; 9:5271-81. [PubMed: 14614009]

13. Summy JM, Gallick GE. Src family kinases in tumor progression and metastasis. Cancer Metastasis Rev. 2003; 22:337-58. [PubMed: 12884910]

14. Posadas EM, Al-Ahmadie H, Robinson VL, et al. FYN is overexpressed in human prostate cancer. BJU Int. 2009; 103:171-77. [PubMed: 18990162]

15. Myoui A, Nishimura R, Williams PJ, et al. C-SRC tyrosine kinase activity is associated with tumor colonization in bone and lung in an animal model of human breast cancer metastasis. Cancer Res. 2003; 63:5028-33. [PubMed: 12941830]

16. Rucci N, Recchia I, Angelucci A, et al. Inhibition of protein kinase c-Src reduces the incidence of breast cancer metastases and increases survival in mice: implications for therapy. J Pharmacol Exp Ther. 2006; 318:161-72. [PubMed: 16627750]

17. Saad F. Src as a therapeutic target in men with prostate cancer and bone metastases. BJU Int. 2009; 103:434-40. [PubMed: 19154462] 
18. Mendiratta P, Mostaghel E, Guinney J, et al. Genomic strategy for targeting therapy in castrationresistant prostate cancer. J Clin Oncol. 2009; 27:2022-29. [PubMed: 19289629]

19. Lee FY, Lombardo L, Camuso A, et al. BMS-354825 potently inhibits multiple selected oncogenic tyrosine kinases and possesses broad-spectrum antitumor activities in vitro and in vivo [abstract 675]. Proc Am Assoc Cancer Res. 2005; 46:159.

20. Lombardo LJ, Lee FY, Chen P, et al. Discovery of $N$-(2-chloro-6-methyl-phenyl)-2-(6-(4-(2hydroxyethyl)-piperazin-1-yl)-2-methylpyrimidin-4-yla-mino)thiazole-5-carboxamide (BMS-354825), a dual Src/Abl kinase inhibitor with potent antitumor activity in preclinical assays. J Med Chem. 2004; 47:6658-61. [PubMed: 15615512]

21. Nam S, Kim D, Cheng JQ, et al. Action of the Src family kinase inhibitor, dasatinib (BMS-354825), on human prostate cancer cells. Cancer Res. 2005; 65:9185-89. [PubMed: 16230377]

22. O'Hare T, Walters DK, Stoffregen EP, et al. In vitro activity of Bcr-Abl inhibitors AMN107 and BMS-354825 against clinically relevant imatinib-resistant Abl kinase domain mutants. Cancer Res. 2005; 65:4500-5. [PubMed: 15930265]

23. Cortes J, O'Brien S, Borthakur G, et al. Efficacy of dasatinib in patients with previously untreated chronic myelogenous leukemia in early chronic phase [abstract 182]. Blood. 2008:112. [PubMed: 17890457]

24. Guilhot F, Apperley J, Kim DW, et al. Dasatinib induces significant hematologic and cytogenetic responses in patients with imatinib-resistant or -intolerant chronic myeloid leukemia in accelerated phase. Blood. 2007; 109:4143-50. [PubMed: 17264298]

25. Hochhaus A, Baccarani M, Deininger M, et al. Dasatinib induces durable cytogenetic responses in patients with chronic myelogenous leukemia in chronic phase with resistance or intolerance to imatinib. Leukemia. 2008; 22:1200-6. [PubMed: 18401416]

26. Ottmann O, Dombret H, Martinelli G, et al. Dasatinib induces rapid hematologic and cytogenetic responses in adult patients with Philadelphia chromosome-positive acute lymphoblastic leukemia with resistance or intolerance to imatinib: interim results of a phaseII study. Blood. 2007; 110:2309-15. [PubMed: 17496201]

27. Walker-Daniels J, Coffman K, Azimi M, et al. Overexpression of the EphA2 tyrosine kinase in prostate cancer. Prostate. 1999; 41:275-80. [PubMed: 10544301]

28. Zeng G, Hu Z, Kinch MS, et al. High-level expression of EphA2 receptor tyrosine kinase in prostatic intraepithelial neoplasia. Am J Pathol. 2003; 163:2271-76. [PubMed: 14633601]

29. Hofer MD, Fecko A, Shen R, et al. Expression of the platelet-derived growth factor receptor in prostate cancer and treatment implications with tyrosine kinase inhibitors. Neoplasia. 2004; 6:50312. [PubMed: 15548358]

30. Park SI, Zhang J, Phillips KA, et al. Targeting SRC family kinases inhibits growth and lymph node metastases of prostate cancer in an orthotopic nude mouse model. Cancer Res. 2008; 68:3323-33. [PubMed: 18451159]

31. Luo FR, Camuso A, McGlinchey K, et al. Evaluation of anti-osteoclastic activity of the novel, oral multi-targeted kinase inhibitor dasatinib (BMS-354825) [abstract B178]. Proceedings of the Molecular Targets and Cancer Therapeutics Meeting. 2005:173.

32. Koreckij T, Nguyen H, Brown LG, Yu EY, Vessella RL, Corey E. Dasatinib inhibits the growth of prostate cancer in bone and provides additional protection from osteolysis. Br J Cancer. 2009; 101:263-68. [PubMed: 19603032]

33. Scher HI, Halabi S, Tannock I, et al. Design and end points of clinical trials for patients with progressive prostate cancer and castrate levels of testosterone: recommendations of the Prostate Cancer Clinical Trials Working Group. J Clin Oncol. 2008; 26:1148-59. [PubMed: 18309951]

34. Shah NP, Kantarjian HM, Kim DW, et al. Intermittent target inhibition with dasatinib $100 \mathrm{mg}$ once daily preserves efficacy and improves tolerability in imatinib-resistant and -intolerant chronicphase chronic myeloid leukemia. J Clin Oncol. 2008; 26:3204-12. [PubMed: 18541900]

35. Therasse P, Arbuck SG, Eisenhauer EA, et al. European Organization for Research and Treatment of Cancer, National Cancer Institute of the United States, National Cancer Institute of Canada. New guidelines to evaluate the response to treatment in solid tumors. J Natl Cancer Inst. 2000; 92:205-16. [PubMed: 10655437] 
36. Lipton A, Cook R, Saad F, et al. Normalization of bone markers is associated with improved survival in patients with bone metastases from solid tumors and elevated bone resorption receiving zoledronic acid. Cancer. 2008; 113:193-201. [PubMed: 18459173]

37. Yu EY, Wilding G, Posadas E, et al. Dasatinib in patients with hormone-refractory progressive prostate cancer: a phase II study [abstract 5156]. J Clin Oncol. 2008; 26:288s.

38. Demers LM, Costa L, Lipton A. Biochemical markers and skeletal metastases. Cancer. 2000; 88:2919-26. [PubMed: 10898335]

39. Brown JE, Cook RJ, Major P, et al. Bone turnover markers as predictors of skeletal complications in prostate cancer, lung cancer, and other solid tumors. J Natl Cancer Inst. 2005; 97:59-69. [PubMed: 15632381]

40. Coleman RE, Major P, Lipton A, et al. Predictive value of bone resorption and formation markers in cancer patients with bone metastases receiving the bisphosphonate zoledronic acid. J Clin Oncol. 2005; 23:4925-35. [PubMed: 15983391]

41. Cook R, Hirsh V, Major PP, et al. Meta-analysis of effects of zoledronic acid on survival in metastatic bone disease: survival in patients with high bone turnover [abstract 20562, page 7293]. J Clin Oncol. 2008:26. [PubMed: 18165637]

42. Cook RJ, Coleman R, Brown J, et al. Markers of bone metabolism and survival in men with hormone-refractory metastatic prostate cancer. Clin Cancer Res. 2006; 12:3361-67. [PubMed: 16740758]

43. Raipar S, Laplanche A, Tournay E, Massard C, Gross-Goupil M, Fizazi K. Prognostic value of urinary N-telopeptide (uNTx) in patients with castration-resistant prostate cancer (CRPC) and bone metastases [abstract 4138, page 2125]. J Clin Oncol. 2008:26. [PubMed: 18165637]

44. Armstrong AJ, Creel P, Turnbull J, et al. A phase I-II study of docetaxel and atrasentan in men with castration-resistant metastatic prostate cancer. Clin Cancer Res. 2008; 14:6270-76. [PubMed: 18829508]

45. Luo FR, Barrett Y, Ji P, et al. Dasatinib (BMS-354825) pharmacokinetics correlate with pSRC pharmacodynamics in phase I studies of patients with cancer (CA180002, CA180003) [abstract 3046, page 1325]. J Clin Oncol. 2006:24.

46. Araujo J, Gallick G, Trudel G, et al. Dasatinib and docetaxel combination treatment for patients with castration-resistant progressive prostate cancer: a phase 1/2 study (CA180-086) [abstract 5061]. J Clin Oncol. 2009; 27:15s. 


\section{Translational Relevance}

Dasatinib is an oral tyrosine kinase inhibitor with potent activity against SRC and SRC family kinases, BCR-ABL, platelet-derived growth factor receptor, and c-KIT. In experimental models, inhibition of SRC has both antitumor effects, directly on prostate cancer cells (proliferation and metastasis), and decreases bone turnover, as shown in this report through declines in urinary $\mathrm{N}$-telopeptide and bone-specific alkaline phosphatase. Thus, dasatinib may have efficacy both against prostate cancer and the negative consequences that the tumor has on bone. As a result of this trial, an ongoing phase III randomized clinical trial is evaluating the effects of the addition of dasatinib to docetaxel on overall survival and skeletal-related events. Should this randomized phase III study show benefit, SRC inhibition may represent a novel therapy with a unique mechanism of action for patients with metastatic castration-resistant prostate cancer who suffer principally from bone morbidity and its complications. 


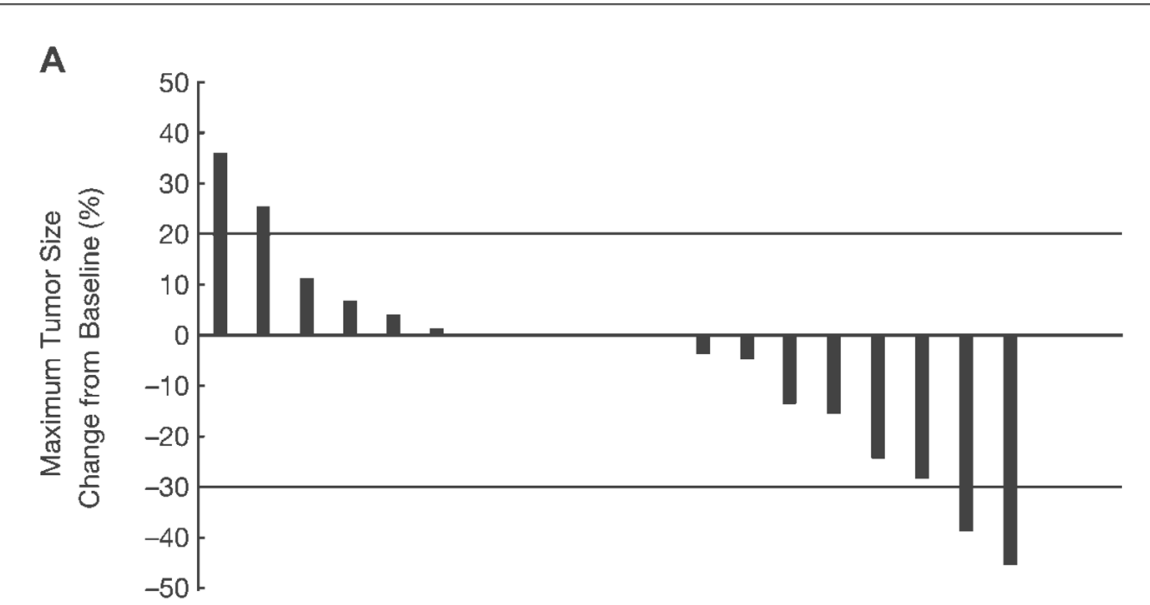

B

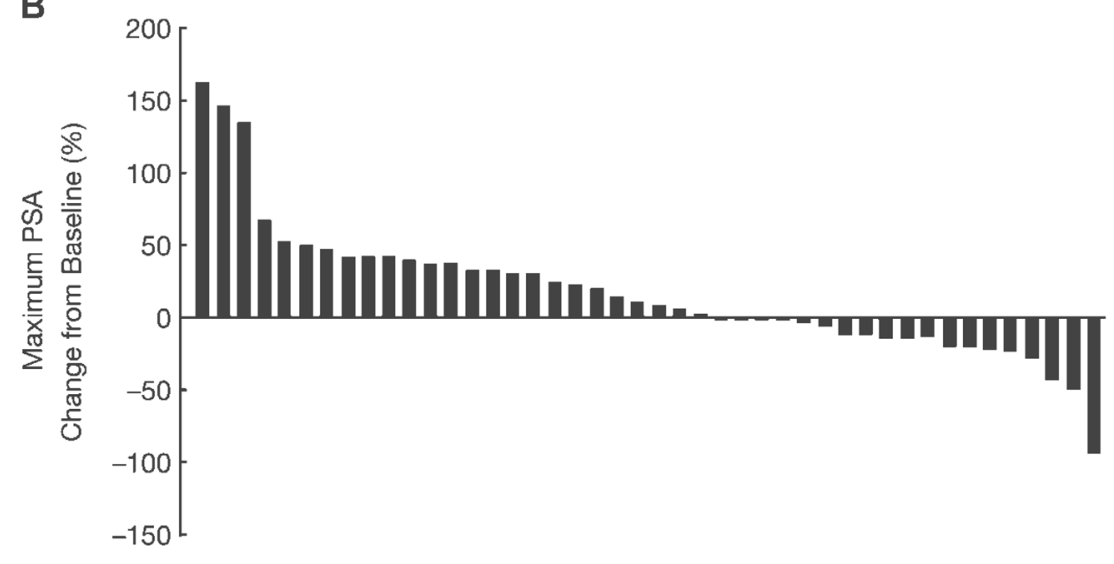

Fig. 1.

$A$, waterfall plot of maximal change (\%) in RECIST-evaluable tumor size from baseline. $B$, waterfall plot of maximal PSA change $(\%)$ from baseline. One patient had a confirmed PSA response, and $79 \%$ of patients had prolonged PSA doubling time. 


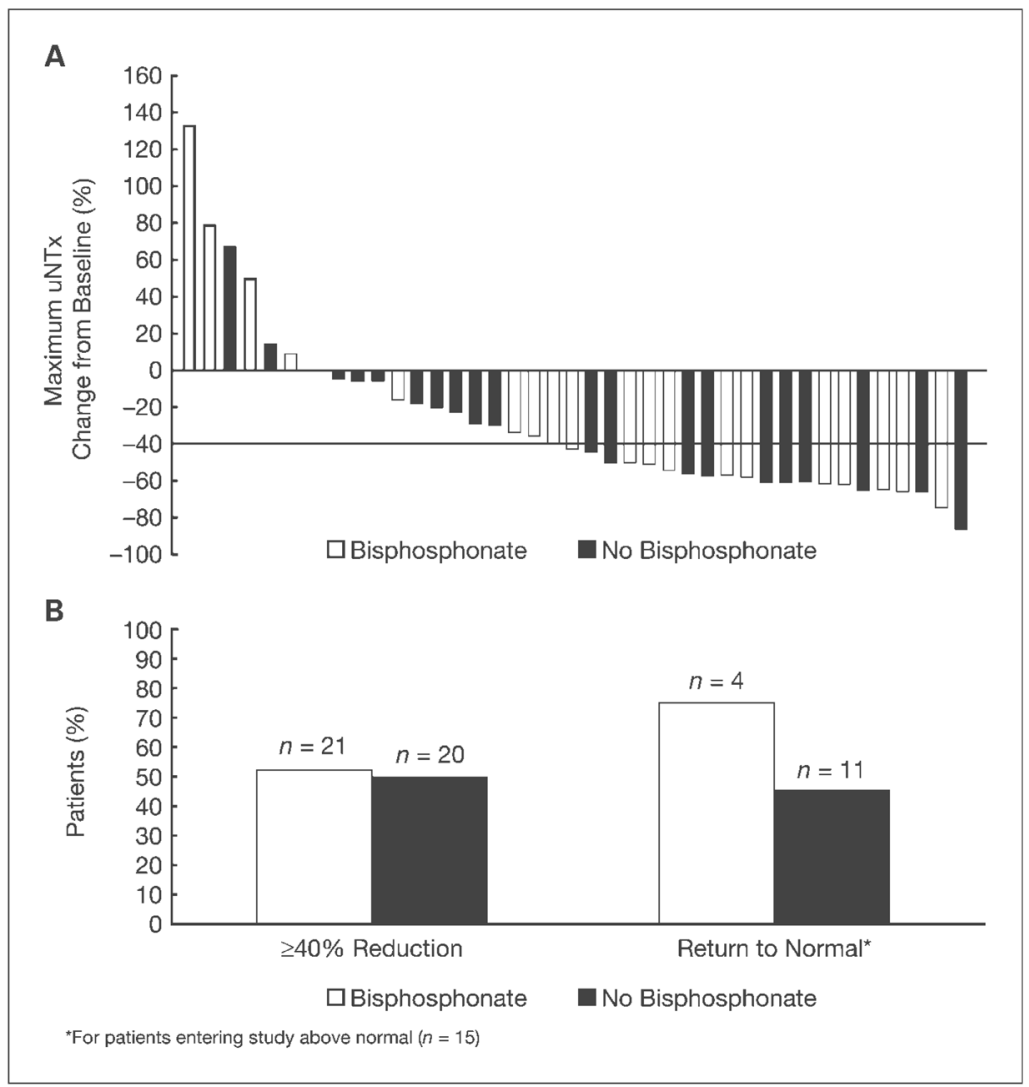

Fig. 2.

$A$, waterfall plot of maximal uNTX change $(\%)$ from baseline. Proportion of patients with $\geq 40 \%$ decrease is reported. $B$, patients achieved $\_40 \%$ decreases of uNTX regardless of whether they were receiving concomitant bisphosphonates. Fifteen patients with baseline uNTX levels above the upper limit of normal normalized on study. 


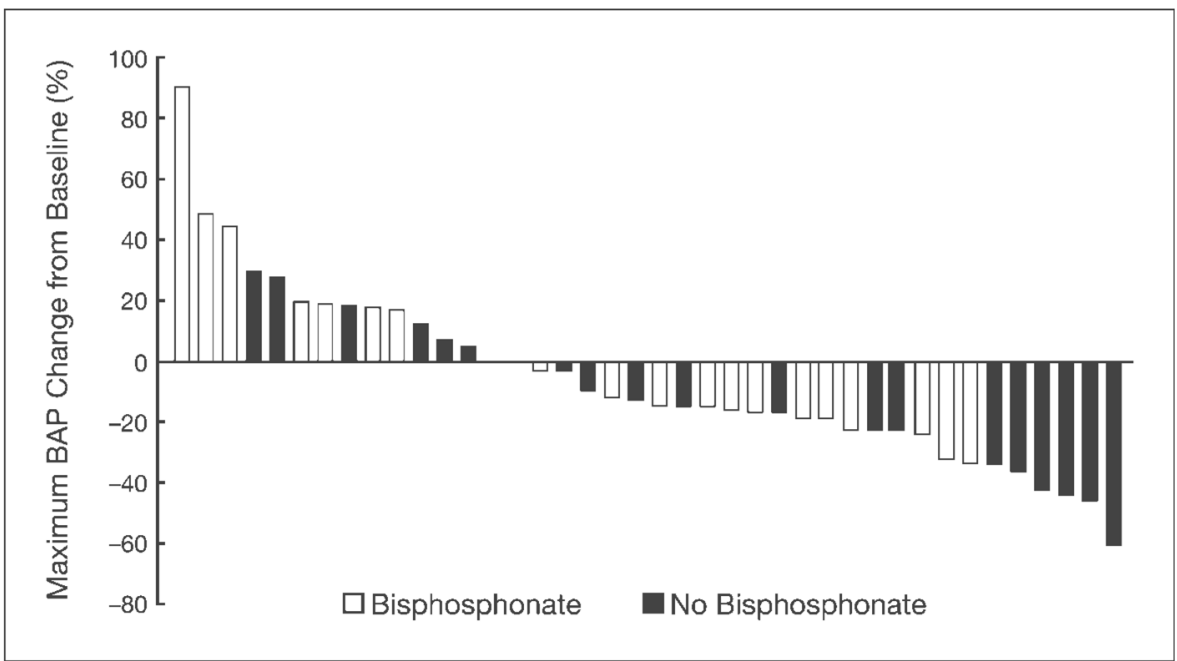

Fig. 3.

Waterfall plot of maximal BAP change (\%) from baseline. 
Table 1

Patient characteristics and demographics

\begin{tabular}{|lc|}
\hline Characteristics & \\
\hline Total no. assessable patients & 47 \\
Median (range) age, y & $70(54-87)$ \\
Age $\ 65$ y, $n(\%)$ & $34(72)$ \\
Race/ethnicity, $n(\%)$ & \\
Caucasian & $45(96)$ \\
Black/African American & $2(4)$ \\
Median (range) time since diagnosis, mo & $52(10-191)$ \\
Eastern Cooperative Oncology Group performance status, $n(\%)$ \\
0 & $28(60)$ \\
1 & $18(38)$ \\
2 & $1(2)$ \\
Prior therapy, $n(\%)$ & \\
Surgery & $31(66)$ \\
Radiotherapy & $30(64)$ \\
Bisphosphonates & $23 *(47.9)$ \\
RECIST-evaluable disease, $n(\%)$ & $25(53)$ \\
Bone metastases, $n(\%)$ & $41(87.2)$ \\
Disease sites, target lesions, $n(\%)$ & $1(2)$ \\
Lymph node & $22(47)$ \\
Pelvis & $3(6)$ \\
Adrenal gland & $1(2)$ \\
Visceral, lung & \\
Visceral, liver & \\
Visceral, diaphragm & \\
& \\
&
\end{tabular}

Includes one patient who started bisphosphonate therapy while on study (protocol violation). 
Table 2

Treatment dosing and discontinuation information

\begin{tabular}{|lc|}
\hline Evaluable population & \\
\hline Total no. assessable patients & 47 \\
Still on treatment at time of analysis, $n(\%)$ & $2(4)$ \\
Discontinued treatment, $n(\%)$ & $45(96)$ \\
$\quad$ Disease progression & $32(68)$ \\
$\quad$ Study drug toxicity & $7(15)$ \\
Adverse event unrelated to study drug & $1(2)$ \\
$\quad$ Subject withdrawal of consent & $4(9)$ \\
$\quad$ Investigator decision to withdrawn subject & $1(2)$ \\
Median (range) duration of dasatinib therapy, mo & $2.86(0.03-13.83)$ \\
Median (range) dasatinib dose, mg/d & $139(90-200)$ \\
$\geq 1$ dose reduction, $n(\%)$ & $16(64)$ \\
$\quad$ Patients initially treated with 100 mg twice daily $(n=25)$ & $11(50)$ \\
Patients initially treated with $70 \mathrm{mg}$ twice daily $(n=22)$ & \\
$\geq 1$ dose interruption, $n$ (\%) & $15(60)$ \\
$\quad$ Patients initially treated with $100 \mathrm{mg}$ twice daily $(n=25)$ & $13(59)$ \\
$\quad$ Patients initially treated with $70 \mathrm{mg}$ twice daily $(n=22)$ & \\
\hline
\end{tabular}


Table 3

Drug-related adverse events occurring in $\geq 10 \%$ of patients

\begin{tabular}{|lcccc|}
\hline Adverse event & All grades $(\boldsymbol{\%})$ & Grade 1 (\%) & Grade 2 (\%) & Grade 3 (\%) \\
\hline Nonhematologic & & & & \\
Diarrhea & 61.7 & 44.7 & 12.8 & 4.3 \\
Pleural effusion & 51.1 & 17.0 & 31.9 & 2.1 \\
Nausea & 46.8 & 31.9 & 12.8 & 2.1 \\
Fatigue & 44.7 & 14.9 & 19.1 & 10.6 \\
Headache & 36.2 & 31.9 & 4.3 & 0 \\
Anorexia & 36.2 & 21.3 & 14.9 & 0 \\
Dyspnea & 36.2 & 14.9 & 14.9 & 6.4 \\
Rash & 34.0 & 23.4 & 10.6 & 0 \\
Asthenia & 27.7 & 10.6 & 12.8 & 4.3 \\
Pericardial effusion & 23.4 & 23.4 & 0 & 0 \\
Peripheral edema & 21.3 & 17.0 & 4.3 & 0 \\
Prolonged QT interval & 19.1 & 17.0 & 2.1 & 0 \\
Body weight decrease & 17.0 & 12.8 & 4.3 & 0 \\
Flushing & 17.0 & 17.0 & 0 & 0 \\
Vomiting & 12.8 & 6.4 & 6.4 & 0 \\
Edema & 10.6 & 6.4 & 0 & 4.3 \\
Flatulence & 10.6 & 8.5 & 2.1 & 0 \\
Arthralgia & 10.6 & 6.4 & 4.3 & 0 \\
Dry skin & 10.6 & 10.6 & 0 & 0 \\
Appetite decrease & 10.6 & 8.5 & 2.1 & 0 \\
Dizziness & 10.6 & 8.5 & 2.1 & 0 \\
Hematologic & & & & 2.1 \\
Anemia & 95.7 & 72.3 & 21.3 & 2.1 \\
Thrombocytopenia & 36.2 & 34.0 & 0 & 2.1 \\
Leukopenia & 25.5 & 14.9 & 6.4 & 4.3 \\
Neutropenia & 19.1 & 12.8 & & \\
\hline
\end{tabular}

There were no grade $4 / 5$ adverse events. 